\title{
Experiences and Challenges Faced During Transportation of Critically Ill Patients
}

\section{Bhagya $\mathbf{S}^{1}$, Kathyayini Vijayalekshmi Revivarman ${ }^{2}$, Dhanasekaran B. $\mathbf{S}^{\mathbf{3}}$, Gireeshkumar K. $\mathbf{P}^{4}$, Sreekrishnan T. $\mathbf{P}^{5}$, Manna Maria ${ }^{6}$}

${ }^{1}$ BSc EMT, ${ }^{2,6}$ MD Resident, ${ }^{3}$ Professor, ${ }^{4} \mathrm{HOD},{ }^{5}$ Assistant Professor, Dept of Emergency Medicine, Amrita Institute of Medical Sciences, Kochi, Kerala

Corresponding Author: Kathyayini Vijayalekshmi Revivarman

\begin{abstract}
Objective: This study is aimed to assess the incidence of major events during transportation of critically ill patients.

Methods: A prospective observational study was conducted in 200 patients during inter hospital transportation from January 2017 to December 2017 at Amrita Institute of Medical Sciences (AIMS), Kochi after fulfilling both inclusion and exclusion criteria. The main objective is to assess the incidence of major events during transportation of critically ill patients. The variables recorded in this study includes age, gender, co-morbidities, airway, breathing, circulation - related events during inter hospital transportation and duration of transportation. Inclusion criteria include critically ill patients in the age group of 18 - 100 years and exclusion criteria include pregnant patients.

Results: On assessing the data of all 200 critically ill patients who had undergone inter hospital transport about $38.5 \%$ patients had major events compromising the circulation .In this study Hypertension (27.5\%) is the more common baseline disease that deteriorated while transportation. $38.5 \%$ of study population had major events compromising the circulation, $12.5 \%$ had major events in breathing and $35.5 \%$ patients had no major events in their airway, breathing and circulation during transportation.

Conclusion: During the transportation of critically ill patients under the monitoring of trained medical personnel $38.5 \%$ of major events occurred in the circulation part. Respiratory issues were presented in $27 \%$ patients (54), of which 30 had desaturation and remaining had tachypnea, which was managed by NIV or Invasive ventilation. 5\% of patients had airway compromise, managed by appropriate basic or advanced airway maneuvers. Hence pre transport checklist and continuous monitoring by an experienced medical personnel is necessary for the prevention of adverse events during transportation.
\end{abstract}

Keywords: Critically ill patients, Interhospital transportation, major events.

\section{INTRODUCTION}

Patient transport is an integral part of the management of critically ill patients. It is often done to improve the existing management of patients. Transport of the critically ill patients may be within the same facility for any diagnostic procedure, or to another facility for advanced care. A wide variety of adverse events have been associated with the transport of critically ill patients, ranging from equipment failure, device removal to haemodynamic instability and death.

The transport of critically ill patients is defined as" the transportation of a patient between medical facilities without interruption of the medical treatment and monitoring due to the underlying disease by means of specific medical ,technical equipment and knowledge with the objective of improved patient care" ${ }^{\text {(1) }}$.

The interhospital transports are increasing due to establishment of regional 
ICU centers, centralization of certain surgical procedures and availability of certain therapeutic interventions. Intra hospital transport rates had increased due to the newer imaging modalities and radiological interventions that cannot be performed at bed side ${ }^{(2)}$. Inta hospital and inter hospital transfer of patients pose major threat to patient safety ${ }^{(2-3)}$. A crucial first step is to determine whether transport is essential. The decision to transfer patient is based on the benefits of care available at another facility against the potential risks involved ${ }^{(4)}$. Several diagnostic procedures (endoscopy, echocardiography, ultrasonography, etc) and therapeutic procedures (tracheostomy, feeding jejunostomy, etc.) can be performed at the bedside, thus avoiding the need for transport. However, an increasing array of investigations or treatment modalities such as computed tomography (CT), digital subtraction angiography and magnetic resonance imaging (MRI) cannot be performed at the bedside and hence the need for intra hospital or inter hospital transport. Critically ill patients are transported to alternate locations to obtain additional care, whether technical, cognitive or procedural, that are not available at the existing location. Despite the inherent risks, transport may be inevitable if the benefits exceed the risks involved. Hence, all personnel dealing with critically ill patients must acquire the technical as well as organisational skills to ensure the safe transport of critically ill patient ${ }^{(6)}$.

The principal aims during the transport of a critically ill patient are to ensure patient safety and to provide the same intensity and quality of care en route, as that being provided in the Intensive Care Unit (ICU). Patient transfer is considered a part of critical care continuum ${ }^{(7)}$. It also involves providing the same intensity of care at a remote location that may not be designed to care for such patients, in a potentially unfavorable and resourcerestricted environment (e.g., MRI suite). Fulfilling this aim requires adequately trained staff, pre-transport assessment and stabilization of patients, planning and organization.Transfer of critically ill patients places tremendous responsibility on the transporting personnel, and in a vulnerable situation in case of patient or equipment related troubleshooting ${ }^{(6)}$. The procedure of transport has been divided into the pre-transport, transport and posttransport phases.

Serious complications were averted when the problem was detected during a pre-transport check. This highlights the need to have checklists that contain systematic checkpoints during all the three phases. A meticulous pretransport preparation and stabilization should be done before transport to prevent any adverse events or deterioration of patients' condition. Pre transport assessment of the airway, breathing, circulation and disability of transported patients and elective intervention help in avoiding any untoward event during transportation. Thus, a safe system not only includes checklists, but also includes audit of performance, root-cause analysis of critical incidents, education and feedback. Barriers to the use of checklists include inadequate knowledge, emergency transport and lengthy, non-user-friendly checklists. Appropriate decision to transfer the patient, pre transfer stabilization and preparation, appropriate mode of transfer, the competency of personnel accompanying the patient, adequate drugs, equipments and monitoring, documentation are the keystones in patient transfer ${ }^{(4)}$.

The patient related major events are assessed in this study to identify major troubleshooters and hence can be prepared for the required interventions beforehand.

\section{METHODOLOGY Inclusion criteria}

Critically ill patients in the age group of 18 - 100years

\section{Exclusion criteria}

Pregnant females 


\section{Materials Required}

Glucometer, cardiac monitor, defibrillator, oxygen cylinder.

\section{Structure, Material And Location}

A prospective observational study was conducted in 200 patients during inter hospital transportation from January 2017 to December 2017 at Amrita Institute of
Medical Sciences (AIMS), Kochi after fulfilling both inclusion and exclusion criteria. The main objective is to assess the incidence of major events during transportation of critically ill patient. The variables recorded include age, sex, comorbidities, duration of transport and events occurred during pre transport, transport and post transport.

\section{RESULTS}

\section{DISTRIBUTION OF MAJOR EVENTS IN A,B \& C}

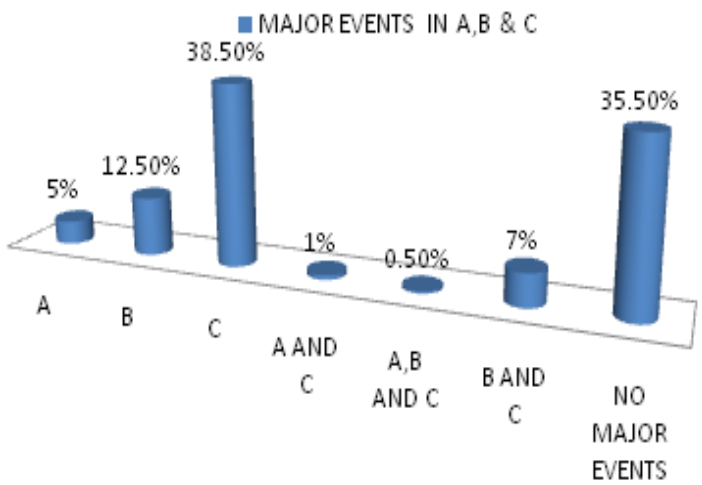

Table:1- Distribution of major events in airway ,breathing and circulation.

\section{Distribution of invasive and non-invasive ventilation}

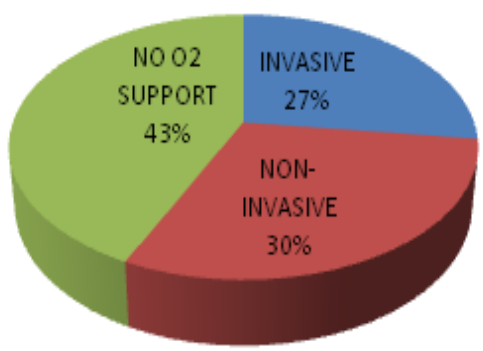

Figure 2: distribution of invasive andnon invasive ventilation

Among 200 patients who had transported, 5\% (10) patients had events compromising airway, $12.5 \% \quad(25)$ interfering breathing, $38.5 \% \quad$ (77) complicating circulation. $7 \%$ (14) had events in both breathing and circulation, $1 \%$ (2) had events in both airway and circulation and $0.5 \%$ (1) had major events in airway, breathing and circulation. $35.5 \%$ (71) patients had no events compromising their airway, breathing or circulation (Figure 1). There were no deaths during the transport. Among 200 patients transported, $27 \%$ (54) patients were on invasive ventilation, $30 \%$ (60) patients were on noninvasive ventilation, while $43 \%$ (86) patients required no ventilator supports (Figure 2). Among 200 patients transported, $12.5 \%$ (25) developed hypotension and required inotropic support. Nor-adrenaline was given to $6.5 \%$ (13), dopamine to $3.5 \%$ (7), Dobutamine to $2 \%$ (4), and adrenaline to $0.5 \%$ (1) (Figure 3). 


\section{DISTRIBUTION OF IONOTROPES}

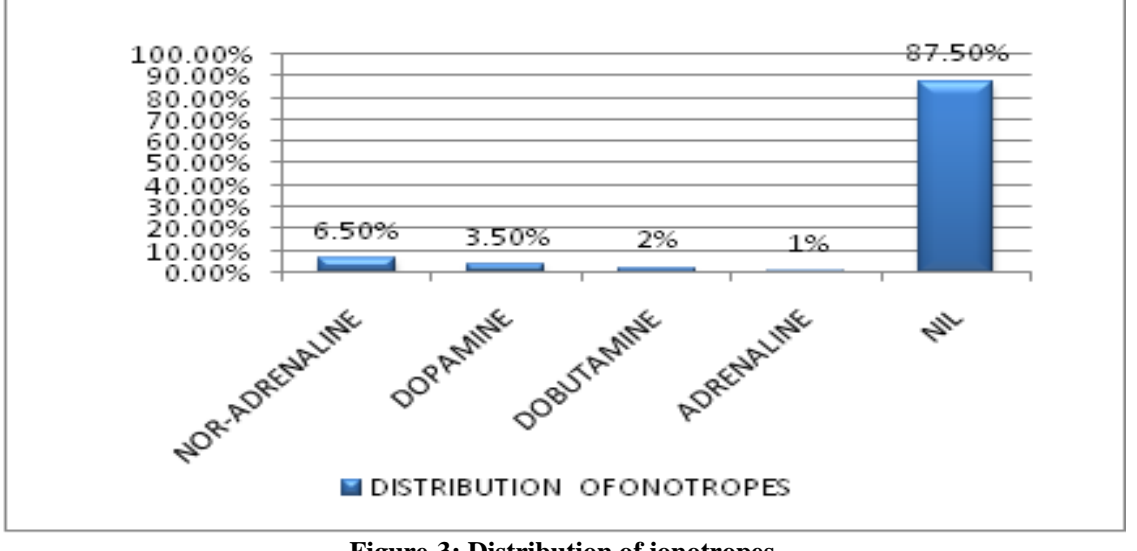

Figure-3: Distribution of ionotropes

\section{DISCUSSION}

The study is a prospective observational study done on 200 patients who had underwent inter hospital transport. The study was done to identify the major events during transportation. Among the 200patients, $61.5 \%$ were males and remaining $38.5 \%$ were females and $35 \%$ of patients were in the age group of $61-80$ years.

Adverse events occurred in $64.5 \%$ of transports. Among the 200 patients who were transported, 5\% (10) had events compromising the airway, $12.5 \%$ (25) had events interfering with breathing, $38.5 \%$ (77) had events complicating circulation. In a study conducted by $\mathrm{C}$. Waydhas adverse events occurred in $70 \%$ of transports and in another study by Lovell et al $31 \%$ of transports had patient related adverse events. The patient related adverse events include change in heart rate, hypotension, hypertension, increased Intracranial Pressure, arrhythmias, change in respiratory rate, hypocapnia, hypercapnia, hypoxia and hypoglycaemia ${ }^{(10)}$.

$15 \%$ (30) patients had desaturation $(\mathrm{SpO} 2<94 \%)$ while the remaining $85 \%$ maintained saturation level of $94-100 \%$. Respiratory issues were presented in $27 \%$ patients (54), of which 30 had desaturation and remaining had tachypnea, which was managed by NIV or Invasive ventilation. Among 200 patients transported, 27\% (54) patients were on mechanical ventilation,
$30 \%$ (60) were on non-invasive ventilation. $5 \%$ of patients had airway compromise managed by appropriate basic or advanced airway maneuvers. In study conducted by Lovell et al 5\% patients had desaturation which was managed by Oxygen support ${ }^{(10)}$.

This study revealed that major events occurred in circulation part of about $38.5 \%$ patients. In the study population, 77 patients had tachycardia, 116 patients had normal heart rate range and 7 patients had bradycardia. Among 200 patients transported, $12.5 \% \quad$ (25) developed hypotension and required inotropic support. Nor-adrenaline was given to $6.5 \%$ (13), dopamine to $3.5 \%$ (7), Dobutamine to $2 \%$ (4), adrenaline $0.5 \%$ (1). In a similar study by Lovell et al hypotension developed in 6 $\%$ patients and requiring inotropic support. $7 \%$ had tachycardia and 3\% had bradycardia which were also managed by the trained transport personnel accompanying the patient ${ }^{(10)}$.

Among 200 patients, $11 \%$ developed hypoglycemia during transportation. The patients who had hypoglycemia were managed with IV fluids $25 \%$ Dextrose or DNS.

Risk factors for the development of patient related complications include high PEEP, history of coronary artery disease, postoperative patients, severe trauma patients, and complex medications or infusions. Inexperienced staff, insufficient preparations, equipment malfunctioning, 
poor communication, long duration of transport also lead to complications in inter hospital patient transfer ${ }^{(4)}$.

The patients transported can develop major events posing threat to the airway, breathing parameters and circulation, requiring interventions. The patients at risk of airway compromise can be stabilized by elective endotracheal intubation.

\section{CONCLUSION}

During the transportation of critically ill patients under the monitoring of trained medical personnel $38.5 \%$ of major events occurred in the circulation part. Respiratory issues were presented in $27 \%$ patients (54), of which 30 had desaturation and remaining had tachypnea, which was managed by NIV or Invasive ventilation. $5 \%$ of patients had airway compromise managed by appropriate basic or advanced airway maneuvers. Hence pre transport checklist and continuous monitoring by an experienced medical personnel is necessary for the prevention of adverse events during transportation. Despite improvements in intra hospital and inter hospital practices, significant risks are still involved. Basic training, good clinical sense and a riskbenefit analysis are currently the only deciding factors.

\section{Acknowledgement: None}

Conflict of Interest: None

Source of Funding: None

Ethical Approval: Approved

\section{REFERENCES}

1. T Kiss, A Bolke, P M Spieth. Interhospital transfer of critically ill patients.Minerva Anestesiologica 2017 October;83(10):11018.

2. J M Droogh,M Smit,J G Zijlstra:Interhospital transfer of critically ill patients; expect surprises. Crit care.2012;16(1):R26

3. Beckmann U, Gillies D M, Berenholtz SM, Wu AM, Pronovost P.:Incidents relating to the intra hospital transfer of critically ill patients.Intensive care Med 2004;30:15791585.

4. A.Kulshresta and J.Singh : Inter - hospital and intra - hospital patient transfer: Recent concepts .Indian J Anaesth.2016 Jul;60(7):451-457.

5. Runcie CJ, Reeve W, Reidy J, et al: Secondary transport of the critically ill adult. Clin Intensive Care 1991; 2:217-225 (c)

6. Burtnyk S: Secondary transportation of critically ill people-Implications for nurses and the need for specialist training. IntensiveCrit Care Nurs 1992; 8:234-239 (c)

7. Venkataraman ST, Orr RA: Intrahospital transport of critically ill patients. Crit Care Clin 1992; 8:525-531 (c)

8. American College of Emergency Physicians. Principles of appropriate patient transfer. Ann Emerg Med 1990; 19:337-338 (d)

9. Kruse D: Interhospital Transfer:. How to prepare your patient. Nursing 1991; 21:41 (c)

10. Waydas C: Intra hospital transport of critically ill patients. Crit Care 1999; 3:R83-R89.

How to cite this article: Bhagya S, Revivarman $\mathrm{KV}$, Dhanasekaran B. S et.al. Experiences and challenges faced during transportation of critically ill patients. Int J Health Sci Res. 2021; 11(7):124-128. DOI: https://doi.org/10.52403/ ijhsr.20210718 